\title{
Reviewer Acknowledgements for Vol. 5, No. 1, March 2017
}

Journal of Agricultural Studies would like to acknowledge the following reviewers for their assistance with peer review of manuscripts for this issue. Many authors, regardless of whether JAS publishes their work, appreciate the helpful feedback provided by the reviewers. Their comments and suggestions were of great help to the authors in improving the quality of their papers. Each of the reviewers listed below returned at least one review for this issue

\section{Reviewers for Volume 5, Number 1}

Eliana Mariela Werbin, National University of Cordoba, Argentinian

Ewa Moliszewska, Opole University, Poland

Pramod Kumar Mishra, Indian Institute of Management Bangalore, India

Sahar Bahmani, University of Wisconsin at Parkside, USA

Sait Engindeniz, Ege University Faculty of Agriculture, Turkey

Gerardo Ojeda, Universidad Nacional de Colombia, Colombia

Hui Guo, University of Georgia, USA

Zakaria Fouad Abdallah, National Research Centre, Egypt

Zhao Chen, Clemson University, US

Chenlin Hu, The Ohio State University, USA

Luisa Pozzo, IBBA of Pisa, Italy

Aftab Alam, Edenworks Inc. New York, USA

Ernest Baafi, CSIR-Crops Research Institute, Ghana

Jeong Hwan, Sejong University, South Korea

Abhishek A. Cukkemane, Bijasu Agri Research Laboratory LLP, India

Soto Caro Ariel Reinaldo, Universidad de Concepción, Chile

Richard Williams

Editor

Journal of Agricultural Studies

Macrothink Institute

5348 Vegas Dr.\#825

Las Vegas, Nevada 89108

United States

Phone: 1-702-953-1852 ext.521

Fax: 1-702-420-2900

Email: jas@macrothink.org

URL: http://jas.macrothink.org 